Check for updates

Cite this: RSC Adv., 2017, 7, 42073

\title{
Poly(lactic-co-glycolic) acid nanoparticles improve oral bioavailability of hypocrellin A in rat
}

\author{
Ling-Yuan Guo, (D) a Shu-Zhen Yan, (D) a Qiang Li, (D) a Qiao Xu, (D) a Xi Lin, (D) a \\ Shan-Shan Qi, (D) a Shu-Qin Yu (D) ${ }^{* b}$ and Shuang-Lin Chen (D) *a
}

Hypocrellin A (HA), a perihydroxylated polycyclic quinone isolated from the fungus of Shiraia bambusicola, exhibits a wide spectrum of biological activities in pre-clinical studies. However, poor water solubility is a major clinical constraint factor of HA, leading to unpredictable bioavailability. In this study, HA-loaded poly (lactic-co-glycolic acid) (PLGA) nanoparticles (PLGA/HA NPs) were prepared by the single-emulsion solvent-evaporation technique. Characterization of PLGA/HA NPs showed that HA was successfully encapsulated on the PLGA. The drug-loading content and encapsulation efficiency (EE) were $7.0 \%$ and $57.5 \%$, respectively. The in vitro release profile demonstrated that HA was released more slowly from nanoparticles in $\mathrm{pH} 1.5$ and $\mathrm{pH} 6.8$ than in $\mathrm{pH}$ 7.4. Furthermore, the highest solubility of PLGA/HA NPs in aqueous solution was approximately 35.67-fold that of native HA ( $\mathrm{n}-\mathrm{HA})$. PLGA/HA NPs had more superior stability compared with n-HA in physiological conditions. In this paper, a sample and sensitive LC-MS/MS method was validated for the first time to quantify HA in rat plasma. The pharmacokinetic parameters and bioavailability of HA between PLGA/HA NPs and n-HA were compared after oral administration in rat. The results implied that the relative bioavailability of PLGA/HA NPs was 2.67-fold that of $n-H A$, and PLGA/HA NPs had a longer half-life. Therefore, these results suggested that PLGAblend nanoparticles improved the solubility, stability and bioavailability of $\mathrm{HA}$, and could become a potential and promising carrier for the oral delivery of $\mathrm{HA}$.

Received 27th April 2017

Accepted 12th August 2017

DOI: $10.1039 / \mathrm{c} 7 \mathrm{ra0} 4748 \mathrm{~g}$

rsc.li/rsc-advances
In addition, HA is a hydrophobic drug, and its solubility must be improved to maximize its potential bioavailability. ${ }^{10}$ One strategy to improve HA solubility is chemical modification. Typical chemical modification techniques included attempted salt formation, complexed with metal ions, and added cosolvents, hydrotropic agents and surfactants. These approaches increase solubility by altering a solvating environment, making it better suited for maintaining hydrophobic solutes in aqueous solution. ${ }^{11}$ Indeed, several research approaches have been reported to enhance HA solubility, including sulfonation of $\mathrm{HA}^{12}$ and chelation of HA with metal ions..$^{13,14}$ However, most of the HA derivatives generated to date have exhibited less PDT efficacy than their parent; those water-soluble derivatives reduced cellular uptake and led to poor photodynamic activity in vitro, storage stability and biocompatibility. ${ }^{15}$

In recent years, nano drug delivery systems (NDDS) have provided remarkable advantages in enhancing water solubility and pharmacological activity of photosensitizers. ${ }^{16-20}$ As a biodegradable polymers, PLGA has been extensively used in NDDS for lower toxicity and excellent loaded-drug efficacy. ${ }^{17,19,20}$ PLGA improved loaded drugs the dispersibility, solubility, and absorption in vitro, and prolonged the half-life time and plasma circulation in vivo of loaded drugs. ${ }^{19,21}$ In addition, PLGA is degraded in the body into nontoxic lactic acid and glycolic acid, which are the normal components of Kreb's cycle, and
${ }^{a}$ College of Life Sciences, Nanjing Normal University, Nanjing 210046, The People's Republic of China. E-mail: chenshuanglin@njnu.edu.cn; Fax: +86 258589 1571; Tel: +862585891571

Jjiangsu Province Key Laboratory for Molecular and Medical Biotechnology College of Life Sciences, Nanjing Normal University, Nanjing 210046, The People's Republic of China. E-mail: yushuqin@njnu.edu.cn; Fax: +86 258589 1265; Tel: +86 2585891265 
subsequently eliminated as carbon dioxide and water without affecting the normal cellular functions. ${ }^{22}$

In previous work, we reported that HA-loaded PLGA nanoparticles (PLGA/HA NPs) improved the photodynamic anticancer effect. ${ }^{4}$ However, developed nanoparticle formulations need a thorough evaluation in terms of pharmacokinetic investigation. In fact, the physicochemical parameters of nanoparticles, such as particle size, hydrophobicity and surface modification, dramatically changed drug pharmacokinetics and impacted drug bioavailability and biodistribution. ${ }^{19}$ On the basis of these factors, in the present study, we further explored the in vitro release, solubility and stability of PLGA/HA NPs. Moreover, a sensitive liquid chromatography tandem mass spectrometry (LC-MS/MS) method with multiple reaction monitoring (MRM) was developed for the first time and validated for the determination of HA in rat plasma. Finally, we compared the pharmacokinetic parameters and bioavailability of HA between PLGA/HA NPs and n-HA after oral administration in rat.

\section{Materials and methods}

\subsection{Chemicals}

HA (98\%) was isolated from the bodies of Shiraia bambusicola. Docetaxel (99.8\%) was obtained from Aladdin Industrial Corporation (Shanghai, China). Poly (D,L-lactic-co-glycolic acid) (PLGA, $\mathrm{L} / \mathrm{G}=50 / 50, M_{\mathrm{W}}=30 \mathrm{kDa}$ ) was obtained from Ji Nan Dai Gang Biomaterial Corporation (Shandong, China). Polyvinyl alcohol (PVA, $M_{\mathrm{W}}=31 \mathrm{kDa}$ ) and dimethyl sulfoxide (DMSO) were obtained from Sigma-Aldrich (MO, USA). Pepsin (1 : 3000) and trypsin $(1: 250)$ were obtained from Solarbio Corporation (Beijing, China). Spectramax M2 Multiscan Spectrum was obtained from Molecular Devices Corporation (USA). Methanol and acetonitrile (HPLC grade) were purchased from Tedia Company (USA). HPLC-grade water produced by MilliQ System (Millipore, Paris, France) was utilized throughout the experiments. All other chemicals were of analytical grade and commercially available.

\subsection{Preparation of PLGA/HA NPs}

PLGA/HA NPs were obtained using the single-emulsion solventevaporation technique previously reported by our group. ${ }^{4}$ Briefly, HA (10 mg) and PLGA (100 mg) were dissolved in acetone $(2.5 \mathrm{~mL})$ for $6 \mathrm{~h}$ at room temperature. The organic phase was rapidly injected into $50 \mathrm{~mL}$ PVA aqueous solution $(0.5 \%, \mathrm{w} / \mathrm{v})$ and homogenized using a high-speed homogenizer (IKA, Germany), resulting in an oil-in-water $(\mathrm{O} / \mathrm{W})$ solution. Next, the residual organic solvent was eliminated by mechanical stirring at $1500 \mathrm{rpm}$ overnight. The particles were then recovered by centrifugation (Eppendorf Centrifuge 5418R, Germany) at $13500 \mathrm{rpm}$ for $30 \mathrm{~min}$ at $4{ }^{\circ} \mathrm{C}$, and washed twice with distilled water to remove the residual drugs and emulsifiers. Lastly, PLGA/HA NPs were added $2 \%$ sucrose as a freeze-dried adjuvant agent to avoid aggregation; PLGA/HA NPs were freeze-dried at $-80{ }^{\circ} \mathrm{C}$ for $48 \mathrm{~h}$ and stored at $-20{ }^{\circ} \mathrm{C}$ in the dark before use.

\subsection{Drug loading efficiency (LE) and encapsulation efficiency} (EE)

The account of HA loading and encapsulation in PLGA/HA NPs was investigated by HPLC. Chromatographic separation was performed on an Agilent Zorbax Extend-C18 column (4.6 $\times$

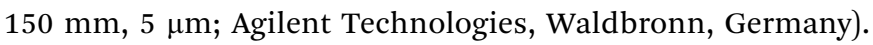
The mobile phase consisted of methanol (A) and water containing $0.1 \%$ formic acid (B) $(90: 10 \mathrm{v} / \mathrm{v})$, pumped isocratic elution at a flow rate of $0.8 \mathrm{~mL} \mathrm{~min}^{-1}$. The sample injection volume was $10 \mu \mathrm{L}$, and the UV detector was used at a wavelength of $464 \mathrm{~nm}$. One milligram of lyophilized PLGA/HA NPs was dissolved in $10 \mathrm{~mL}$ of methanol aimed to extract HA. The samples in methanol were ultrasonically mixed for $15 \mathrm{~min}$ to leach out HA entirely. Then, samples were centrifuged at $13500 \mathrm{rpm}$ for $30 \mathrm{~min}$, and supernatant was collected. An aliquot of $10 \mu \mathrm{L}$ was used for HPLC analysis. A calibration curve was established by HA standard solutions. The calibration curve had good linearity $\left(r^{2}=0.999\right)$, ranging from $0.75-$ $100 \mu \mathrm{g} \mathrm{mL}{ }^{-1}$. The amount of HA loaded and encapsulated in PLGA/HA NPs was expressed as LE (1) or EE calculated as follows (2):

$$
\begin{aligned}
\text { Loading efficiency }(\%)= & \frac{\text { weight of } \mathrm{HA} \text { in nanoparticles }}{\text { weight of nanoparticles }} \\
& \times 100
\end{aligned}
$$

Encapsulation efficiency $(\%)=\frac{\text { weight of HA in nanoparticles }}{\text { weight of total HA }}$ $\times 100$

\subsection{In vitro release profile}

The release of HA from PLGA/HA NPs was carried by dissolving PLGA/HA NPs in artificial gastric ( $\mathrm{pH}$ 1.5), intestinal ( $\mathrm{pH} 6.8$ ) and blood $(\mathrm{pH}$ 7.4) media. Those suspensions were kept at $37{ }^{\circ} \mathrm{C}$ and $100 \mathrm{rpm}$. The release of HA from PLGA/HA NPs was conducted by the dialysis membrane method. PLGA/HA NPs $(10 \mathrm{mg}$ ) were divided in $2 \mathrm{~mL}$ of distilled water and sealed in a dialysis membrane bag (cut off $M_{\mathrm{W}}=12 \mathrm{kDa}$, Millipore, France). The dialysis bag containing nanoparticles was immersed in $100 \mathrm{~mL}$ artificial gastric ( $\mathrm{pH}$ 1.5), intestinal ( $\mathrm{pH}$ 6.8) and blood ( $\mathrm{pH} 7.4$ ) media with $0.5 \%(\mathrm{w} / \mathrm{v})$ Tween-80 in a conical flask, followed by constant shaking at a speed of $100 \mathrm{rpm}$ at $37^{\circ} \mathrm{C}$. At a determined time, $1 \mathrm{~mL}$ of release medium was withdrawn to analyse. The release medium was completely replaced with the same volume of fresh buffer solution. The released HA was measured by HPLC as mentioned above. All assays were carried out in triplicate.

The drug release rate (RR) was calculated according to the following formula (3):

$$
\begin{aligned}
\text { Drug release } \operatorname{rate}(\%)= & \frac{\mathrm{HA} \text { released amount }}{\text { total amount of HA in nanoparticles }} \\
& \times 100
\end{aligned}
$$




\subsection{Solubility study and stability}

To verify the solubility of n-HA and PLGA/HA NPs, excessive samples (n-HA and PLGA/HA NPs) were dissolved and dispersed into $10 \mathrm{~mL}$ double distilled water at $25 \pm 2{ }^{\circ} \mathrm{C}$, then acutely blended $30 \mathrm{~s}$ every $5 \mathrm{~min}$. After $30 \mathrm{~min}$, samples were taken out and filtered through a $0.22 \mu \mathrm{m}$ Millipore membrane. The filtrate was diluted an appropriate number of times, and the absorbance was detected by UV-vis spectrophotometer at $464 \mathrm{~nm}$. The n-HA and PLGA/HA NPs solubility was calculated by standard curve and dilution times. The chemical stability of HA was determined by exposing both n-HA and PLGA/HA NPs to physiological conditions, and the remaining HA present in the media was measured. Specifically, the n-HA and PLGA/HA NPs suspensions in artificial gastric ( $\mathrm{pH}$ 1.5), intestinal ( $\mathrm{pH}$ 6.8) and blood (pH 7.4) media were kept at $37{ }^{\circ} \mathrm{C}$ and $100 \mathrm{rpm}$. At designated time points $(0,1,2,4,7,10,12 \mathrm{~h})$, samples were removed and detected using Multiscan Spectrum at $464 \mathrm{~nm}$. All of the analyses were expressed as the mean \pm standard deviation (S.D.) of three replicates.

\subsection{Animal}

Sprague-Dawley rats were kept in an environmentally controlled breeding room and fed with standard laboratory food and water for two weeks before starting the experiments. The experimental protocol were conformed to the principles of the National Institutes of Health guide for the care and use of Laboratory Animals, (NIH publications no. 8023, revised 1978) and approved by the Animal Care and Institutional Animal Ethics Committee of Nanjing Normal University.

\subsection{Chromatograph system and conditions for HA quantification}

The LC-MS/MS analysis was conducted in positive ion ESI mode on a Waters Quattro Micro System equipped with a triplequadrupole mass spectrometer connected to a liquid chromatograph (Waters Alliance). Chromatographic separation was performed on an Agilent Zorbax Extend-C ${ }_{18}$ column $(4.6 \times 150$ mm, $5 \mu \mathrm{m}$; Agilent Technologies, Waldbronn, Germany). The column temperature was maintained at $28{ }^{\circ} \mathrm{C}^{23}$ The mobile phase consisted of methanol (A), and water containing $0.1 \%$ formic acid (B) (90:10 v/v) was used for isocratic elution at

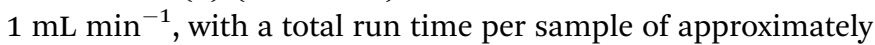
$5 \mathrm{~min}$. The injection volume was $10 \mu \mathrm{L}$.

Mass spectrometric detection was performed on a Waters Quattro Micro triple-quadrupole mass spectrometer equipped with an electrospray ionization (ESI) interface. Both the analyte HA and internal standard (IS) docetaxcel were monitored under positive ion-multiple reaction monitoring (+MRM) mode with transitions of $m / z 547.1 \rightarrow 529$ for HA, and $m / z 830.7 \rightarrow 549.4$ for IS, respectively. Other parameters of the mass spectrometer were as follows: spray voltage, $3250 \mathrm{~V}$; vaporizer temperature, $300{ }^{\circ} \mathrm{C}$; and capillary temperature, $350^{\circ} \mathrm{C}$. Argon was used as the collision gas for collision-induced dissociation (CID), $3.2 \times$ $10^{-3}$ mbar; solvent off gas flow rate, $400 \mathrm{~L} \mathrm{~h}^{-1}$; and gas curtain gas velocity, $50 \mathrm{~L} \mathrm{~h}^{-1}$. The auto sampler cooler was maintained at $4{ }^{\circ} \mathrm{C}$.
The data were acquired and processed using Analyst version MassLynx software (Version 4.0). For quantification, the peak area ratios of the target ions of the HA were compared to those of the IS with weighted $1 / X^{2}$ (where $X=$ drug concentration) least squares calibration curves in which the peak area ratios of the calibration standards were plotted versus their concentrations.

\subsection{Preparation of stock, standard and quality control samples}

Stock solutions of $\mathrm{HA}\left(100 \mu \mathrm{g} \mathrm{mL}^{-1}\right)$ and IS docetaxel $\left(500 \mu \mathrm{g} \mathrm{mL} \mathrm{m}^{-1}\right)$ were dissolved in methanol. The IS working solution was diluted with methanol to $2.5 \mu \mathrm{g} \mathrm{mL}{ }^{-1}$. Standard solutions of HA in rat plasma were prepared by spiking with an appropriate volume of the serially diluted stock solutions, giving final concentrations of 15, 50, 100, 200, 500, 1 000, 1 500, and $2000 \mathrm{ng} \mathrm{mL}{ }^{-1}$ for HA.

Quality control (QC) samples were prepared from blank plasma at high, medium and low concentrations of 1500,500 ,

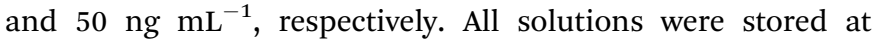
$-20{ }^{\circ} \mathrm{C}$ prior to analysis. In addition, all the samples containing the calibration samples, QC samples and plasma samples were processed and prepared according to the procedure described as follows.

\subsection{Plasma sample preparation}

The $100 \mu \mathrm{L}$ of heparinized plasma sample (or containing $10 \mu \mathrm{L}$ HA blank plasma) was aliquoted into a $1.5 \mathrm{~mL}$ polypropylene tube and mixed with $10 \mu \mathrm{L}$ IS solution $\left(2.5 \mu \mathrm{g} \mathrm{mL}^{-1}\right)$ and $50 \mu \mathrm{L}$ acetic acid. Then, $500 \mu \mathrm{L}$ of acetonitrile was added, and the solution was vortexed for $5 \mathrm{~min}$. Next, the solution was ultrasonically mixed for $10 \mathrm{~min}$ and then centrifuged at $16000 \mathrm{rpm}$ for $10 \mathrm{~min}$. After centrifugation, $400 \mu \mathrm{L}$ of the supernatant was transferred to a clean vial and dried with concentrator plus (Eppendorf 5305, Germany). The residue was re-dissolved with $100 \mu \mathrm{L}$ methanol: $\mathrm{H}_{2} \mathrm{O}(50: 50, \mathrm{v} / \mathrm{v})$, and a $10 \mu \mathrm{L}$ aliquot of the sample was injected into LC-MS/MS for analysis.

\subsection{Method validation}

Methods were validated for specificity, linearity and sensitivity, accuracy and precision, extraction recovery and matrix effect according to the US Food and Drug Administration (FDA) guidance.

2.10.1 Specificity. The specificity of the method was evaluated by comparing the chromatograms of blank plasma from six different rats with the corresponding plasma samples spiked with HA and IS, as well as real samples collected from treated rats after intravenous (i.v.) administration for $5 \mathrm{~min}$.

2.10.2 Linearity and sensitivity. Calibration curves were constructed by plotting the peak-area ratio of HA to the IS versus the concentrations of the analytes. It was achieved from eightpoint calibration curves covering a concentration range from 15 to $2000 \mathrm{ng} \mathrm{mL}^{-1} \mathrm{HA}$ in plasma samples, using a weighted least-squares linear regression (the weighting factor, $1 / X^{2}$ ). The lower limit of quantification (LLOQ) was determined based on at least 10 times the signal-to-noise ratio at which the precision 
(expressed by relative standard deviation, RSD) and accuracy (calculated by relative error, RE) were lower than $20 \%$.

2.10.3 Accuracy and precision. The intra-day accuracy and precision were investigated by determining six replicates of high, medium and low QC samples at concentrations of 1 500, 500 and $50 \mathrm{ng} \mathrm{mL^{-1 }}$ on the same day. The inter-day accuracy and precision were studied by determining three levels of QC samples on three consecutive days. The accuracy calculated as follows (4):

Accuracy $(\%)$
$=\frac{\text { mean observed concentration }- \text { spiked concentration }}{\text { spiked concentration }} \times 100$

2.10.4 Extraction recovery and matrix effect. Extraction recovery of HA was determined by comparing the peak area of the HA spiked and extracted from blank plasma with those of extracted blank plasma spiked with the $\mathrm{HA}$ at the same concentration levels. Recoveries were determined at three QC concentrations for plasma $(n=6)$. Matrix effects were calculated by the mean peak area ratios of blank plasma samples spiked with HA after extraction divided by the injected working solution with $\mathrm{HA}$ at the same QC concentrations. The extraction recovery (5), matrix effects calculated as follows (6):

Extraction recovery $(\%)$

$$
=\frac{\text { mean peak area of extracted samples }}{\text { mean peak area of un-extracted samples }} \times 100
$$

Matrix effect $(\%)$

$$
=\frac{\text { mean peak area of un-extracted samples }}{\text { mean peak area in neat standard solutions }} \times 100
$$

2.10.5 Stability. The stability of HA was tested by analysing six replicates of HA plasma samples at three QC concentrations under different conditions: $6 \mathrm{~h}$ exposure at room temperature, three freeze-thaw cycles at $-20{ }^{\circ} \mathrm{C}$, and one week storage at $-20{ }^{\circ} \mathrm{C}$. The stock solution stability of the HA was evaluated using three QC levels samples against freshly spiked solutions. If assay values were within acceptable limits of accuracy $( \pm 15 \%$ $\mathrm{RE})$ and precision ( $\leq 15 \% \mathrm{RSD})$, samples were considered stable.

\subsection{Pharmacokinetic study of HA and PLGA/HA NPs in rat}

Eighteen Sprague-Dawley rats (male and female) with body weight of 200-220 $\mathrm{g}$ were divided into three groups $(n=6)$. The first group was i.v. administered $\mathrm{n}-\mathrm{HA}\left(4 \mathrm{mg} \mathrm{kg}^{-1}\right)$ in $5 \%$ glucose aqueous solution, the second group was orally administered n-HA (40 mg kg-1) prepared in $0.5 \%$ carboxymethyl cellulose sodium (CMC-Na) to form a suspension, and the third group was orally administered PLGA/HA NPs (containing HA $12.5 \mathrm{mg} \mathrm{kg}{ }^{-1}$ ) in aqueous solution. All rats were fasted overnight before drug administration. Blood samples $(300 \mu \mathrm{L})$ were drawn from the orbital vein into heparinized centrifuge tubes under anaesthesia at 0.0833 (only for first group), 0.25, 0.5, 1, 2,
$3,4,6,8,10,12,24$, and $48 \mathrm{~h}$ after dosing. The blood samples were centrifuged at $4000 \mathrm{rpm}$ for $10 \mathrm{~min}$. The supernatant liquor was collected and stored at $-20{ }^{\circ} \mathrm{C}$ until used for analysis.

\subsection{Pharmacokinetics data analysis}

All in vitro analyses were expressed as the mean \pm standard deviation (S.D.) and repeated three times. Furthermore, the in vivo pharmacokinetics results were described as the mean \pm S.D. of six replicates. The pharmacokinetics parameters including the area under curve (AUC), $t_{1 / 2}$ elimination, volume of distribution $\left(V_{\mathrm{d}}\right)$ and apparent clearance (CL) calculated using WinNonlin software (Pharsight 6.2, NC, USA) with compartmental analysis. Statistical analysis was performed using Graph Pad Prism (Graph Pad Inc, USA). Statistical comparison of mean values was evaluated by ANOVA test, and the results were considered statistically significant if $P$-value $<$ 0.05 .

\section{Results and discussion}

\subsection{Preparation of PLGA/HA NPs}

In our study, PLGA/HA NPs were prepared by emulsion solvent evaporation technique. As the linker in PLGA/HA NPs, PVA is a widely used stabilizer for PLGA polymers. The particle size of these nanoparticles was within the range of 20-200 $\mathrm{nm}$ reported by Qi et al. ${ }^{4}$ The drug loading content of the nanoparticles was $7.0 \%$, and encapsulation efficiency of the nanoparticles was $57.5 \%$. These characteristics did not differ significantly from previous experimental results. Particle size, loading and encapsulation efficiency influenced drug solubility, stability and dispersibility. Moreover, properties of PLGA, including concentration, molecular weight, and volume ratio, were related to drug delivery system. ${ }^{24}$

\subsection{In vitro release profile}

Several mechanisms were reported that were responsible for the drug release from the nanoparticles, including surface and bulk erosion, desorption and diffusion. ${ }^{25}$ Most of the drug-loaded nanoparticles presented a biphasic release pattern: there was an initial burst release followed by a sustained release of the drug. However, low molecular weight PLGA NPs have also been reported to exhibit a zero-order release profile, particularly with hydrophobic drug molecules. ${ }^{26}$ In our study, the dialysis bag diffusion technique was used to study the in vitro drug release of HA from PLGA/HA NPs in artificial gastric ( $\mathrm{pH} 1.5)$, intestinal ( $\mathrm{pH}$ 6.8) and blood ( $\mathrm{pH}$ 7.4) biological conditions over a period of $144 \mathrm{~h}$ at $37^{\circ} \mathrm{C}$. The cumulative percentage release of HA from PLGA/HA NPs is depicted in Fig. 1. The in vitro drug release kinetics showed a biphasic release only in $\mathrm{pH} 7.4$, with a fast rate of HA release to $29.3 \%$ within the initial $3 \mathrm{~h}$. However, we observed that HA release in $\mathrm{pH} 1.5$ and $\mathrm{pH} 6.8$ exhibited zeroorder release profiles. HA release was sustained in $\mathrm{pH} 1.5, \mathrm{pH}$ 6.8 and $\mathrm{pH} 7.4$ to a total of approximately $4.7 \%, 18.8 \%$ and $45 \%$, respectively. At the early stage, the rapid release rate of HA from the nanoparticles may be due to partial absorption or precipitation on the surface of the nanoparticles. Later, HA showed 


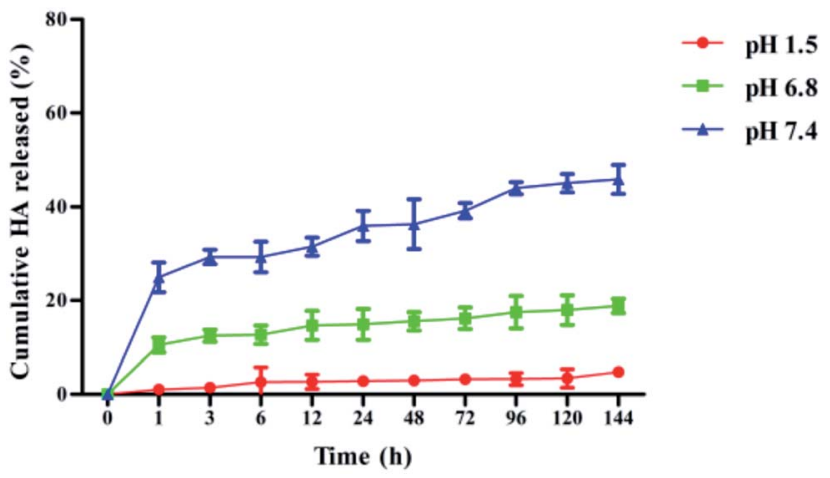

Fig. 1 Release rates of HA from PLGA nanoparticles in vitro in artificial gastric ( $\mathrm{pH}$ 1.5), intestinal $(\mathrm{pH}$ 6.8) and blood $(\mathrm{pH}$ 7.4) biological conditions over a period of $144 \mathrm{~h}$ (mean \pm S.D., $n=3$ ).

a sustained slow release rate that mainly because diffuse from being encapsulated in nanoparticles.

\subsection{Solubility and stability study}

To investigate the solubility of HA and PLGA/HA NPs, samples were dissolved in double distilled water, forming a supersaturated solution. The results in Fig. 2 showed that the dissolved PLGA/HA NPs formed a red aqueous solution, while the n-HA was hydrophobic and hardly dissolved in aqueous solution, with a slightly visible soluble compound. The PLGA/HA NPs aqueous solution dissolved much more than did n-HA. The highest solubility of PLGA/HA NPs and n-HA was $643 \mu \mathrm{g} \mathrm{mL}{ }^{-1}$ and $18.03 \mu \mathrm{g} \mathrm{mL}{ }^{-1}$, respectively. The solubility of PLGA/HA NPs in aqueous solution was approximately 35.67 -fold that of n-HA. In general, poorly water-soluble drugs often show low bioavailability when administered orally, because the absorption of the drugs in the gastrointestinal tract can usually be a rate-limiting step. ${ }^{27}$ Therefore, it is important to enhance drug solubility for improved bioavailability. PLGA could increase the drug solubility and adhesion by forming the stable and amorphous nanoparticles to eliminating the oversaturation of the difference between the particle size of nanoparticles. ${ }^{11,27}$ Moreover, PLGA polymer was commonly used to develop smaller particles with narrow poly dispersity. ${ }^{28}$ We used PLGA as the

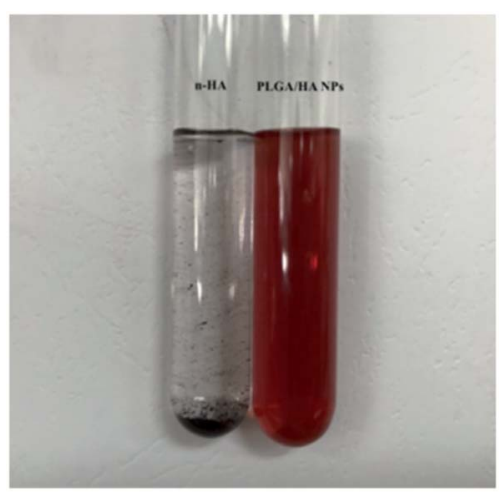

Fig. 2 Solubility of $n-H A$ and PLGA/HA NPs in aqueous solution. nano-carrier because it could distinctly encapsulate drugs into the pore of PLGA to increase hydrophobic drug water solubility. Instability and degradability of drug molecules in physiological $\mathrm{pH}$ conditions hindered drug delivery to target tissue. ${ }^{29}$ To study the stability properties of HA, n-HA and PLGA/HA NPs were incubated in artificial gastric ( $\mathrm{pH}$ 1.5), intestinal (pH 6.8) and blood (pH 7.4) media. As shown in Fig. 3, HA was stable in $\mathrm{pH}$ 1.5 with the change of time, but rapid degradation of n-HA occurred in physiological $\mathrm{pH} 6.8$ and $\mathrm{pH}$ 7.4. Nevertheless, PLGA/HA NPs were more stable under the same conditions, especially under $\mathrm{pH}$ 1.5. Consequently, as a drug carrier, PLGA improved the stability of HA in physiological $\mathrm{pH}$ conditions. PLGA protected the encapsulated HA against enzymatic and hydrolytic biodegradation, thereby preventing HA from breaking down from the gastrointestinal acid. ${ }^{30,31}$

\subsection{Method development}

3.4.1 Selection of IS. In LC-MS/MS analysis, it is important to select a suitable compound for the analyte and serve as an internal standard. Four types of compounds were investigated: 1,8-dihydroxy anthraquinone, lafutidine, $p$-hydroxy benzoic acid butyl ester and docetaxel. However, 1,8-dihydroxy anthraquinone, lafutidine, $p$-hydroxy benzoic acid butyl ester presented low recovery in plasma compared to HA with the same plasma preparation methods. In our study, docetaxel is a stable and commercialized compound with similar mass spectrometric, chromatographic condition and extraction recovery as HA. Thus, docetaxel is the best selection of IS for HA, and it is helpful when a significant matrix effect appeared.

3.4.2 Optimization of the mass condition and chromatographic condition. Given that rat plasma is a complex matrix, a sensitive and stable LC-MS/MS condition was first optimized to quantify HA in plasma samples. First, the standard solution of HA and the IS were injected into the mass spectrometer. The ionization of HA and IS in positive ion and negative ion modes was performed on an ESI ion source and compared in terms of the intensity of produced ions. Finally, HA and IS were analysed in positive ion mode, for the intensity was stronger. In addition, the MRM mode was selected for its better sensitivity and specificity relative to the single ion monitoring (SIM) mode. Thus, the parameters of MS/MS were optimized for the two MRM transitions of $m / z 547.1 \rightarrow 529$ for HA Fig. (4A), and $m / z 830.7$ $\rightarrow 549.4$ for IS Fig. (4B), respectively. The satisfactory resolution of HA and IS was achieved with methanol and water containing $0.1 \%$ formic acid $(90: 10 \mathrm{v} / \mathrm{v})$ with gradient elution at flow rate of $1.0 \mathrm{~mL} \mathrm{~min}^{-1}$ on an Agilent Zorbax Extend-C18 column (4.6 $\times 150 \mathrm{~mm}, 5 \mu \mathrm{m}$ ). Under the present chromatographic condition, the run time of each sample was $5 \mathrm{~min}$, and the retention time was $3.38 \mathrm{~min}, 1.78 \mathrm{~min}$ for HA and IS, respectively.

3.4.3 Sample preparation. To attain clean samples with elimination of endogenous interferences, different extraction procedures were conducted, such as solid phase extraction (SPE), liquid-liquid extraction (LLE) and protein precipitation (PP). The SPE method and LLE method were evaluated for extraction of HA and IS, and undeniably produced the cleanest extracts with high sensitivity, but resulted in lower extraction 

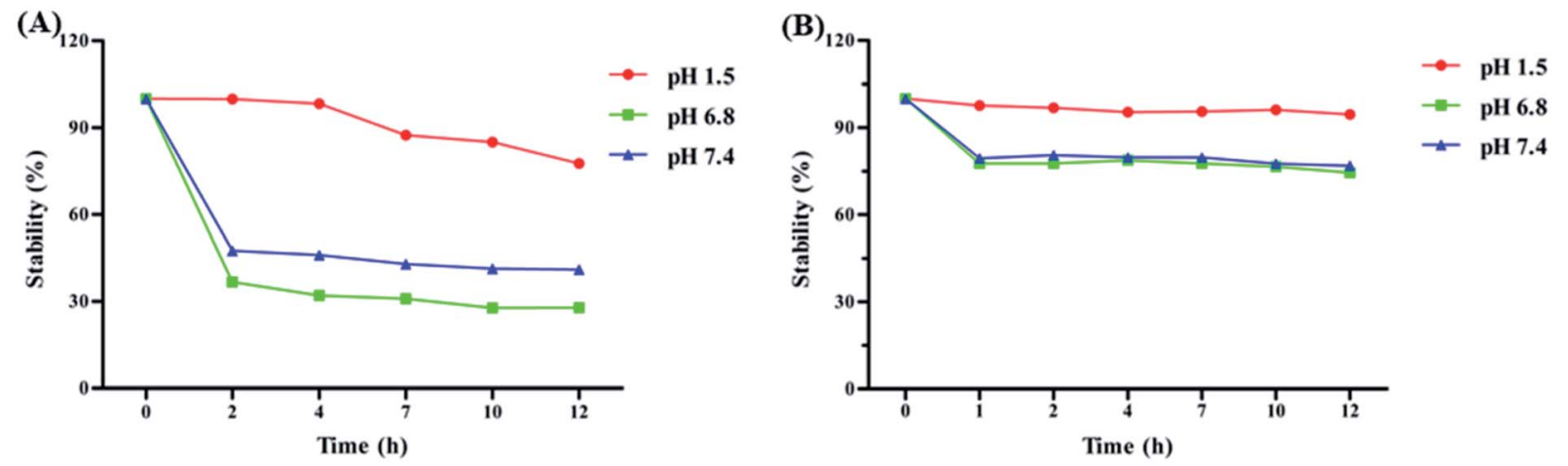

Fig. 3 The stability of $n-H A(A)$ and PLGA/HA NPs (B) in artificial gastric $(\mathrm{pH} 1.5)$, intestinal $(\mathrm{pH} 6.8)$ and blood $(\mathrm{pH} 7.4)$ biological condition at $37^{\circ} \mathrm{C}$ (mean \pm S.D., $n=3$ ).

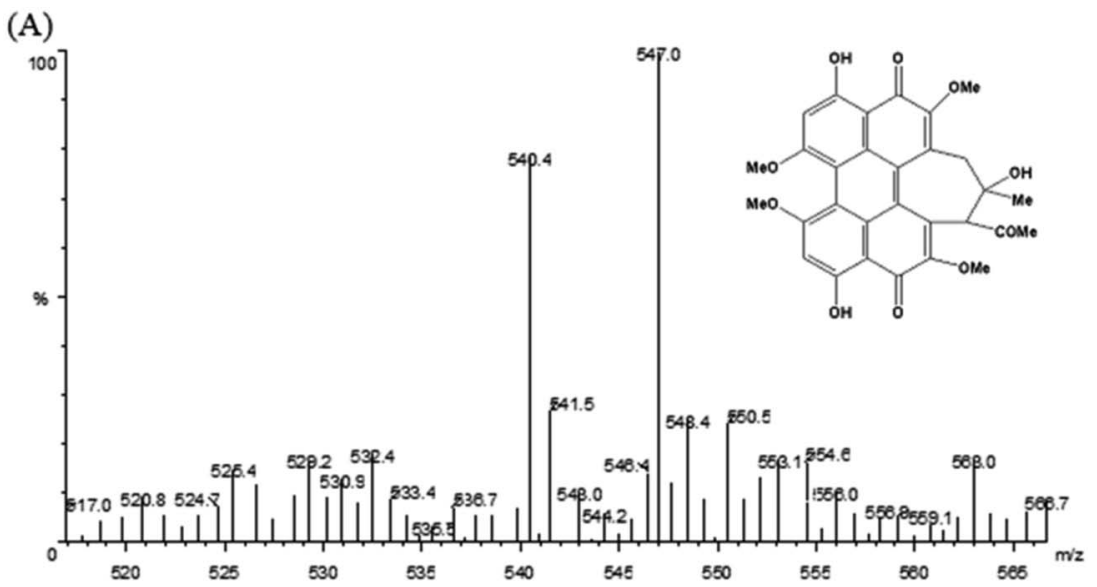

(B)

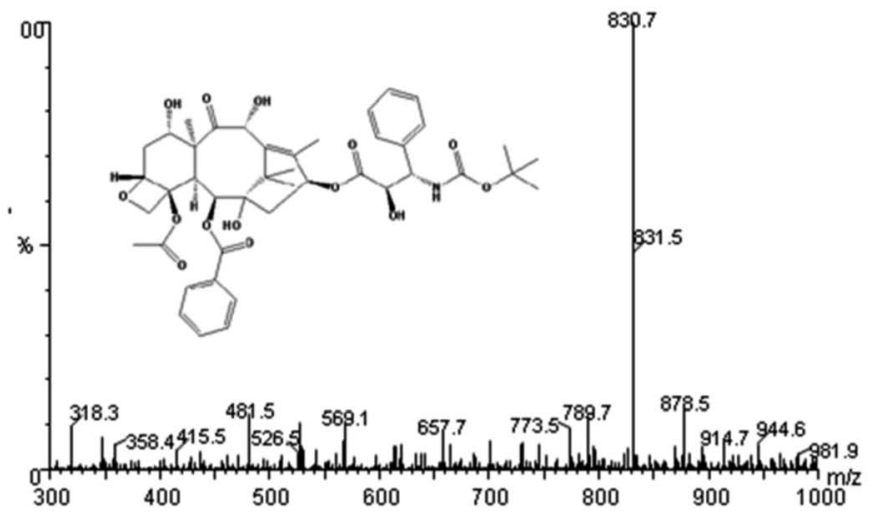

Fig. 4 Product ion mass spectra of HA (A) and IS docetaxel (B) in positive mode.

recovery of HA. However, the PP method was the fastest and simplest method resulting in a clean extract. The PP method also resulted in the high extraction recovery of HA and IS. Acetonitrile was adopted for sample preparation because it offered lower background noise and favourable separation. Indeed, acetic acid was also tested to achieve the maximum recovery of HA. In our study, $10,20,30,40$, and $50 \mu \mathrm{L}$ of acetic acid were tested. Finally, an acetic acid volume of $50 \mu \mathrm{L}$ was the best selection for HA and IS recovery.

\subsection{Method validation}

3.5.1 Specificity. The specificity study aimed to investigate whether endogenous substances in plasma would interfere with the analysis of HA and IS. As shown in Fig. 5, this test included a blank plasma sample (Fig. 5A), plasma containing HA and IS 
(Fig. 5B), and a plasma sample obtained after i.v. administration for $5 \mathrm{~min}$ (Fig. 5C). The results suggested that no endogenous constituents were eluted at the retention time of HA (3.48 $\mathrm{min}$ ) or IS (1.78 $\mathrm{min})$, and the total run time was $5 \mathrm{~min}$.

3.5.2 Linearity and sensitivity. In the current analytical method, the calibration curves contained eight points, covering a concentration range from 15 to $2000 \mathrm{ng} \mathrm{mL} \mathrm{m}^{-1}$, in rat plasma with correlation coefficient $R^{2}>0.9986$ for HA. The LLOQ (signalto-noise ratio $>10$ ) of HA in this method was $10 \mathrm{ng} \mathrm{mL}{ }^{-1}$.

3.5.3 Accuracy and precision. The intra-day and the interday accuracy and precision date of HA in biological matrices are presented in Table 1 . The intra-day and the inter-day precision (RSD, \%) values were $\leq 10.5 \%$ and $\leq 15 \%$, respectively. The intra-day and the inter-day accuracy (\%) values were in the range of $93.27-98.87 \%$ and $94.6-98.87 \%$, respectively. All these results indicated that the method was reproducible and reliable for the determination of HA in rat plasma.
3.5.4 Extraction recovery and matrix effect. The extraction recovery and matrix effect results of HA are summarized in Table 1 . The extraction recovery of HA from low, medium, and high QC samples was in the range from $102 \pm 0.28$ to $110 \pm$ $0.15 \%$. The matrix effect of HA was from $95 \pm 0.05$ to $99.5 \pm$ $0.22 \%$, which was considered negligible or insignificant matrix effects.

3.5.5 Stability. The stability of HA in plasma was evaluated under different conditions (short-term, freeze/thaw, long term stability). The results are shown in Table 2. HA was stable in rat plasma and processed samples under different storage conditions.

\subsection{Pharmacokinetic study}

The validated LC-MS/MS method was first applied to the pharmacokinetic study of HA in plasma after oral administration (40 mg kg $\mathrm{kg}^{-1}$ ) of $\mathrm{n}$-HA (first group), i.v. administration (4 mg $\mathrm{kg}^{-1}$ ) of $\mathrm{n}-\mathrm{HA}$ (second group) and oral administration
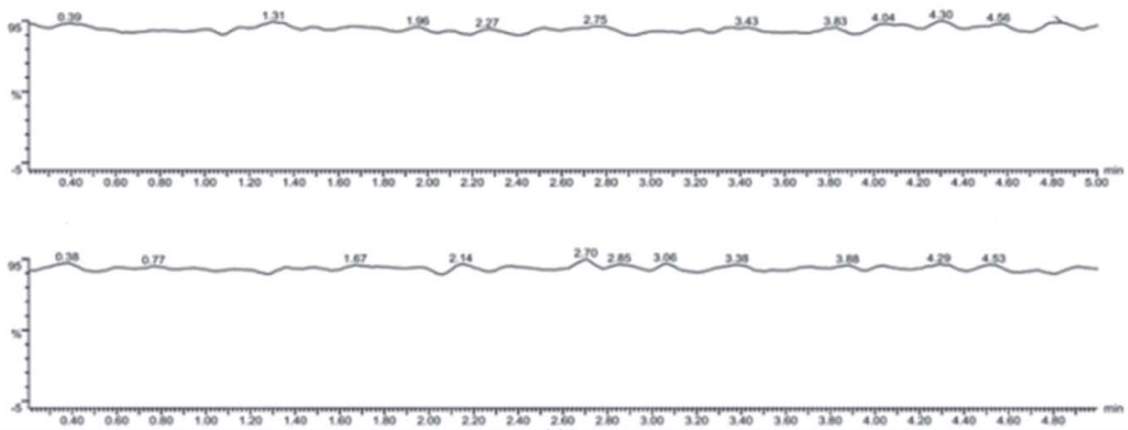

(A)
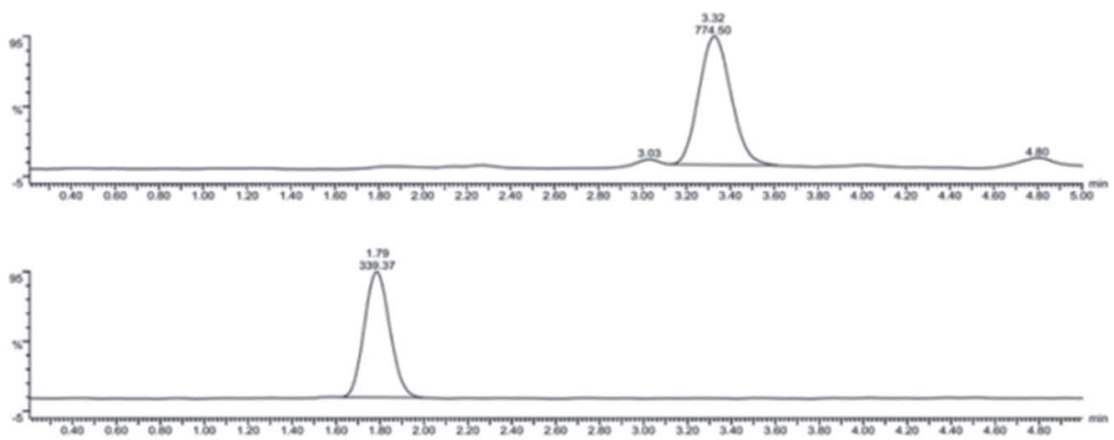

(B)
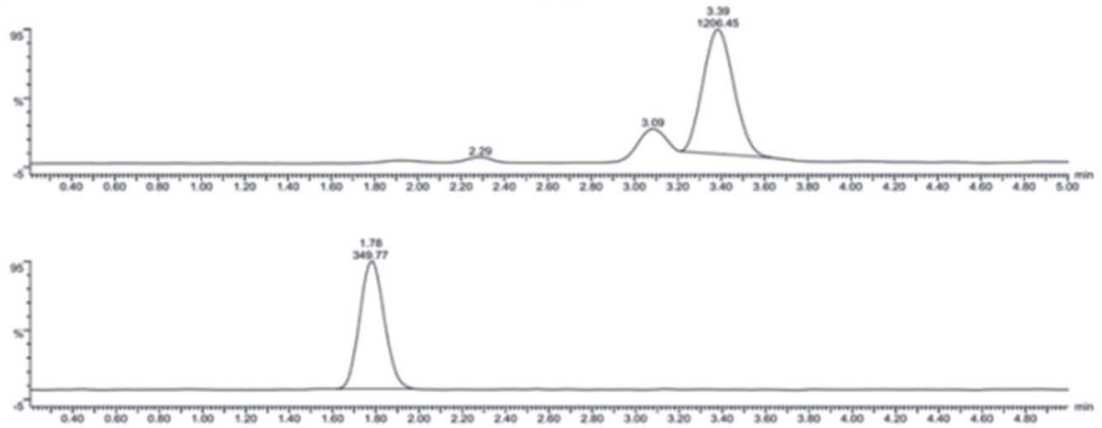

(C)

Fig. 5 Representative MRM chromatograms of HA and IS in plasma: a blank plasma sample (A); a blank plasma sample spiked with HA and IS (B); and a plasma sample collected after i.v. administration $5 \mathrm{~min}(\mathrm{C})$. 
Table 1 Precision, accuracy, extraction recovery and matrix effect for HA in rat plasma $(n=3)$

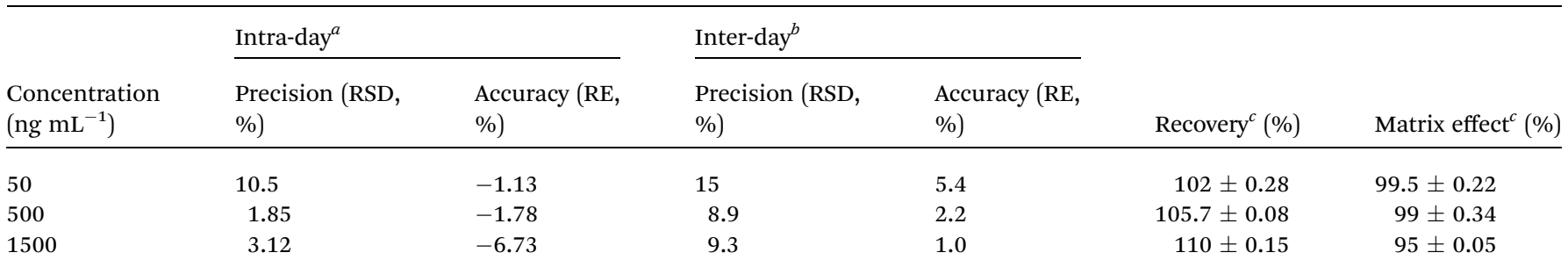

${ }^{a}$ Plasma samples were analyzed three times on the same day. ${ }^{b}$ Plasma samples were run on each of three validation. ${ }^{c}$ Values reported as mean \pm S.D.; $P<0.05$.

Table 2 The results of bench top stability, freeze-thaw stability, long term stability of HA in rat plasma $(n=3)$

\begin{tabular}{llr}
\hline & $\begin{array}{l}\text { Nominal concentration } \\
\text { in QC samples } \\
\left(\mathrm{ng} \mathrm{mL}^{-1}\right)\end{array}$ & $\begin{array}{l}\text { Measured mean } \\
\text { concentration in } \\
\text { stability QC } \\
\text { samples }^{a}\left(\mathrm{ng} \mathrm{mL} \mathrm{mL}^{-1}\right)\end{array}$ \\
\hline Bench top stability & 50 & $55.0 \pm 0.13$ \\
(room temperature, 6 h) & 500 & $491.1 \pm 0.02$ \\
& 1500 & $1399.3 \pm 0.05$ \\
Freeze-thaw stability & 50 & $48.5 \pm 0.05$ \\
$\left(\right.$ after 3 cycles, $\left.-20^{\circ} \mathrm{C}\right)$ & 500 & $511.3 \pm 0.02$ \\
& 1500 & $1548.1 \pm 0.11$ \\
Long term stability & 50 & $40.3 \pm 0.03$ \\
$\left(7\right.$ days, $\left.-20^{\circ} \mathrm{C}\right)$ & 500 & $407.0 \pm 0.02$ \\
& 1500 & $1192.2 \pm 0.06$ \\
${ }^{a}$ Values reported as mean \pm S.D.; $P<0.05$. &
\end{tabular}

(12.5 $\mathrm{mg} \mathrm{kg}^{-1}$ ) of PLGA/HA NPs (third group). The mean HA plasma concentration-time profiles were expressed by a twocompartment model with a weight of $1 / C^{2}$ in Fig. 6 . The relevant pharmacokinetic parameters are shown in Table 3.

The plasma concentration of the first group and the third group were determined in $48 \mathrm{~h}$, while the second group could not be determined after $12 \mathrm{~h}$. The maximum concentration $\left(C_{\max }\right)$ of the three groups was $110.3 \pm 0.274,337.12 \pm 66.28$ and $56 \pm 0.0233 \mathrm{ng} \mathrm{mL}^{-1}$, respectively. $T_{\max }$ of $\mathrm{n}$-HA was shorter than that of PLGA/HA NP after oral administration group $(1.03 \pm 0.274 \mathrm{~h}$ and $2.822 \pm 1.083 \mathrm{~h})$, respectively. The delayed $T_{\max }$ demonstrated a relatively sustained release of HA in PLGA NPs. The $V_{\mathrm{d}}$ of the three groups was $141.87 \pm 37.92,17.01 \pm$ 4.413 and $182.31 \pm 13.475 \mathrm{~L}$, respectively, implying that oral administration of n-HA and PLGA/HA NPs increased the volume of distribution compared to n-HA after i.v. administration. The CL of three groups was $3.771 \pm 1.852,0.203 \pm 0.203$ and $0.578 \pm 0.489 \mathrm{~L} \mathrm{~h}^{-1}$, respectively. These results implied that the elimination rate of HA was low. The CL of the oral administration group was 18.5-fold lower than that of the i.v. administration group.

Recently, PLGA drug delivery systems were developed to offer important advantages in the bioavailability of drugs. In our study, the terminal elimination half-life $\left(t_{1 / 2 \beta}\right)$ and bioavailability of the three groups differed significantly. The $t_{1 / 2 \beta}$ of the three groups was $6.649 \pm 0.0058,2.564 \pm 0.0943$ and
$18 \pm 0.303 \mathrm{~h}$, respectively. The results showed that PLGA/HA NPs had low clearance and long half-life compared with n-HA in rat. PLGA/HA NPs prolonged the absorption phase and decreased the distribution, metabolism and obvious controlled release of HA from the nanoparticles. However, this finding was not consistent with previous results. $\mathrm{Gu}$ et al. reported HAliposome $t_{1 / 2 \beta}$ was 9.85 h. ${ }^{1}$ Pharmacokinetic parameters were altered depending upon the carriers and animal models. In addition, PLGA is frequently used for drug carriers because it may increase the residency time of the sustained release formulation and enhance the drug absorption due to intimacy of contact with the epithelium cells. ${ }^{21} \mathrm{AUC}_{0-t}$ values of the three groups were $1158.2 \pm 171.01,991.67 \pm 219.4$ and $969.69 \pm$ $230.24 \mathrm{ng} \mathrm{h} \mathrm{mL} \mathrm{m}^{-1}$, respectively. The absolute bioavailability of HA was significantly increased from 11.68 to $31.30 \%$. The relative bioavailability of PLGA/HA NPs was approximately $267 \%$ that of HA. The bioavailability of PLGA/HA NPs increased 2.67-fold compared to n-HA.

To date, few studies have reported the bioavailability of HA; only the pharmacokinetic parameters of hypericin, which has an analogue structure of HA, was reported. A study by Schulz et al. showed the poor solubility and bioavailability of hypericin. ${ }^{32}$ In our study, the results demonstrated that PLGA used for HA delivery by oral administration increased HA bioavailability. Therefore, PLGA could potentially be applied to increase the bioavailability of other hydrophobic compounds.

According to experiment results, PLGA contributed to the improved solubility of HA and facilitated HA transport in vivo. In

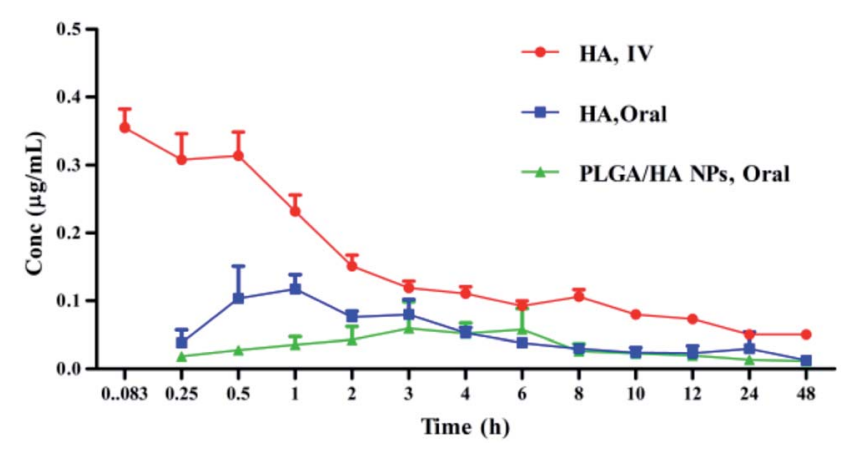

Fig. 6 Plasma mean concentration-time curves of HA after i.v. administration of $4 \mathrm{mg} \mathrm{kg}^{-1}$, oral administration of $40 \mathrm{mg} \mathrm{kg}^{-1}$, and PLGA/HA NPs after oral administration of $12.5 \mathrm{mg} \mathrm{kg}^{-1}(n=6)$. 
Table 3 Pharmacokinetic parameters of $n-H A$, PLGA/HA NPs oral administration and n-HA after i.v. administration in rat $(n=6)^{a}$

\begin{tabular}{llll}
\hline Parameters & n-HA oral & n-HA i.v. & PLGA/HA NPs oral \\
\hline Dose $\left(\mathrm{mg} \mathrm{kg}^{-1}\right)$ & 40 & 4 & 12.5 \\
$C_{\max }\left(\mathrm{ng} \mathrm{mL} \mathrm{mL}^{-1}\right)$ & $110.3 \pm 0.274$ & $337.12 \pm 66.28$ & $56 \pm 0.0233$ \\
$T_{\max }(\mathrm{h})$ & $1.03 \pm 0.274$ & - & $2.822 \pm 1.0835$ \\
$t_{1 / 2 \mathrm{a}}(\mathrm{h})$ & $0.1245 \pm 7.0158$ & - & $0.8797 \pm 0.8148$ \\
$t_{1 / 2 \alpha}(\mathrm{h})$ & $0.9732 \pm 0.5887$ & $2.2814 \pm 3.545$ & $2.553 \pm 0.1228$ \\
$t_{1 / 2 \beta}(\mathrm{h})$ & $6.649 \pm 0.0058$ & $991.67 \pm 219.4$ & $18 \pm 0.303$ \\
$\mathrm{AUC}\left(\mathrm{n}_{0-t}\left(\mathrm{~h} \mathrm{~mL}^{-1}\right)\right.$ & $1158.2 \pm 171.01$ & $17.01 \pm 4.413$ & $969.69 \pm 230.24$ \\
$V_{\mathrm{d}}(\mathrm{L})$ & $141.87 \pm 37.92$ & $0.578 \pm 0.489$ & $182.31 \pm 13.475$ \\
$\mathrm{CL}\left(\mathrm{L} \mathrm{h} \mathrm{h}^{-1}\right)$ & $3.771 \pm 1.852$ & - & $0.203 \pm 0.203$ \\
$F_{\mathrm{a}}(\%)$ & 11.68 & - & 31.30 \\
$F_{\mathrm{r}}(\%)$ & - & & 267
\end{tabular}

${ }^{a}$ Values reported as mean \pm S.D. $(n=6)$. $C_{\max }$ : peak concentration; $T_{\max }$ : time to reach peak concentration; $t_{1 / 2 \alpha}:$ mean distribution half-life; $t_{1 / 2 \beta}$ : mean elimination half-life; $\mathrm{AUC}_{0-t}$ : area under the plasma concentration-time curve; $V_{\mathrm{d}}$ : apparent volume of distribution; CL: clearance; $F$ : oral bioavailability; $P<0.05$.

addition, PLGA protected HA from degradation under physiological conditions and enhanced its stability. All of these advantages favoured improved HA bioavailability. Because of the strong attraction of the particles to the negatively charged mucosa of the intestine responsible for a longer residence time in the intestine, mucoadhesive nanoparticles improve the bioavailability of poorly absorbed drugs. ${ }^{33}$ Nanoparticles have a plethora of advantages over other delivery systems, including the ability to protect drugs from gastrointestinal degradation, prolong systemic circulation, reduce fed/fasted variable absorption, and control drug release. ${ }^{34}$ Therefore, it is expected that with all the aforementioned advantages, the bioavailability of orally delivered drugs in nano form will be improved. However, the mechanism by which PLGA/HA NPs improved HA bioavailability was not detected, and further research is necessary.

\section{Conclusions}

In our study, PLGA/HA NPs have been successfully prepared. PLGA/HA NPs had superior solubility and stability compared with n-HA in physiological conditions. In pharmacokinetic study, a rapid, specific, selective, and reproducible LC-MS/MS method was first developed and validated for the determination of HA in rat plasma. The method was successfully applied to analyse plasma drug concentrations following oral administration of n-HA, PLGA/HA NPs and i.v. administration of n-HA. The pharmacokinetic parameters revealed that PLGA/HA NPs enhanced bioavailability of HA and prolonged HA systemic circulation. Moreover, the improved water solubility of HA was the main reason for its increased bioavailability. Conclusively, encapsulating hydrophobic HA on PLGA polymer will become a promising method for enhanced HA solubility, stability and improved bioavailability.

\section{Conflicts of interest}

There are no conflicts to declare. The authors alone are responsible for the content and writing of this article.

\section{Acknowledgements}

This study was supported by the National Natural Science Foundation of China (Project No. 81673214) the National Key Technology Research and Development Program of the Ministry of Science and Technology of China (Project No. 2012BAD36B0502) and the Priority Academic Program Development of Jiangsu Higher Educational Institutions.

\section{Notes and references}

1 Z. Wang, Y. He, C. Huang, J. Huang, Y. Huang, J. An and Y. Gu, J. Photochem. Photobiol., B, 1999, 70, 773-780.

2 J. Zhang, E. Cao, J. Li, T. Zhang and W. Ma, J. Photochem. Photobiol., B, 1998, 43, 106-111.

3 A. P. Castano, T. N. Demidova and M. R. Hamblin, Photodiagn. Photodyn. Ther., 2005, 2, 91-106.

4 S. Qi, X. Lin, M. Zhang, S. Yan, S. Yu and S. Chen, RSC Adv., 2014, 4, 40085.

5 Z. Diwu, Photochem. Photobiol. Sci., 1995, 61, 529-539.

6 M. J. Fehr, S. L. Carpenter, Y. Wannemuehler and J. W. Petrich, Biochemistry, 1996, 34, 15845-15848.

7 Y. Su, J. Sun, S. Rao, Y. Cai and Y. Yang, J. Photochem. Photobiol., B, 2011, 103, 29-34.

8 G. Ma, S. I. Khan, M. R. Jacob, B. L. Tekwani, Z. Li, D. S. Pasco, L. A. Walker and I. A. Khan, Antimicrob. Agents Chemother., 2004, 48, 4450-4452.

9 S. Xia, Chin. Sci. Bull., 1981, 26, 1040.

10 L. Zhou, J. Zhou, C. Dong, F. Ma, S. Wei and J. Shen, Dyes Pigm., 2009, 82, 90-94.

11 M. Thommes, D. R. Ely, M. T. Carvajal and R. Pinal, Mol. Pharmaceutics, 2011, 8, 727-735.

12 Y. Hu, J. An and L. Jiang, J. Photochem. Photobiol., B, 1993, 17, 195-201.

13 Y. Hu, J. An and L. Jiang, J. Photochem. Photobiol., B, 1994, 22, 219-227.

14 J. Zhou, J. Liu, S. Xia, X. Wang and B. Zhang, J. Phys. Chem. B, 2005, 109, 19529-19535. 
15 J. Zhou, S. Xia, J. Chen, X. Wang and B. Zhang, Chem. Commun., 2003, 12, 1372-1373.

16 Z. Wei, J. An and L. Jiang, J. Photochem. Photobiol., B, 1996, 33, 73-78.

17 Z. Wang, H. Ying, L. Cui, J. Zhang, W. Wang and L. Liu, Chin. J. Clin., 2012, 6, 1443-1446.

18 Y. Qiu, L. U. An and P. Sun, China Surfactant Deterg. Cosmet., 2011, 41, 422-425.

19 N. M. Khalil, T. C. do Nascimento, D. M. Casa, L. F. Dalmolin, A. C. de Mattos, I. Hoss, M. A. Romano and R. M. Mainardes, Colloids Surf., B, 2013, 101, 353-360.

20 J. L. Italia, D. K. Bhatt, V. Bhardwaj, K. Tikoo and M. N. Kumar, J. Controlled Release, 2007, 119, 197-206.

21 Z. Panagi, A. Beletsi, G. Evangelatos, E. Livaniou, D. S. Ithakissios and K. Avgoustakis, Int. J. Pharm., 2001, 221, 143-152.

22 X. Xie, Q. Tao, Y. Zou, F. Zhang, M. Guo, Y. Wang, H. Wang, Q. Zhou and S. Yu, J. Agric. Food Chem., 2011, 59, 9280-9289.

23 X. Gu, J. Zhou, Y. Feng, S. Wei, X. Wang and B. Zhang, J. Instrum. Anal., 2005, 24, 130-132.

24 C. Wischke and S. P. Schwendeman, Int. J. Pharm., 2008, 364, 298-327.
25 D. A. Norris, N. Puri and P. J. Sinko, Adv. Drug Delivery Rev., 1998, 34, 135-154.

26 S. Hariharan, V. Bhardwaj, I. Bala, J. Sitterberg, U. Bakowsky and M. N. V. R. Kumar, Pharm. Res., 2006, 23, 184.

27 M. Sugimoto, T. Okagaki, S. Narisawa, Y. Koida and K. Nakajima, Int. J. Pharm., 1998, 160, 11-19.

28 I. Bala, S. Hariharan and M. N. Kumar, Crit. Rev. Ther. Drug Carrier Syst., 2004, 21(5), 387-422.

29 C. Mohanty and S. K. Sahoo, Biomaterials, 2010, 31, 65976611.

30 E. Fattal, S. Pecquet, P. Couvreur and A. Andremont, Int. J. Pharm., 2002, 242, 15-24.

31 W. He, S. W. Horn and M. D. Hussain, Int. J. Pharm., 2007, 334, 173-178.

32 H. U. Schulz, M. Schürer, D. Bässler and D. Weiser, Arzneim. Forsch., 2005, 55, 15-22.

33 L. C. Simon, R. W. Stout and C. Sabliov, Nanobiomedicine, 2016, 3, 1-10.

34 L. Plapied, N. Duhem, A. D. Rieux and V. Préat, Curr. Opin. Colloid Interface Sci., 2011, 16, 228-237. 